\title{
Star Formation History of the Disk of the Large Magellanic Cloud
}

\author{
J. S. Gallagher and Andrew A. Cole
}

Dept. Astron., U. Wisconsin, Madison, WI 53706, USA

\section{J. Holtzman}

Dept. Astron., New Mexico State U., Las Cruces, NM 88003, USA

\section{T. Smecker-Hane}

Dept. Phys. \& Astron., U. California, Irvine, CA 92697, USA

\begin{abstract}
New optical observations of field stars in the Large Magellanic Cloud from the ground and with the Hubble Space Telescope provide the basis for investigations of the history of field star formation in our nearest galactic neighbor. Results from WFPC2 photometry of field stars indicate that the stellar initial mass function for stars around $1 M_{\odot}$ is similar to that in the solar neighborhood. Long-term variations occurred in the average $S F R$ with enhanced activity in the past 2-4 Gyr, a fallow period about 2-10 Gyr in the past, and an initial phase of astration $\sim 12 \mathrm{Gyr}$ ago. The recent increase in field star formation is less than that for the birth of compact star clusters; the cluster formation rate does not give a complete evolutionary picture.
\end{abstract}

\section{Introduction}

As the nearest galaxy the LMC provides an excellent opportunity to quantitatively test our ideas about the evolution of complex astrophysical systems; including stellar evolution, characteristic of star cluster populations, distribution of metals, importance of bars as dynamical sub-systems, and effects from proximity to the giant Milky Way spiral galaxy (Westerlund 1997). This brief review focuses on the history of the stellar populations in the field of the LMC disk. Since stars may be captured in the LMC bar, this component could have a different history which is not yet properly explored. Understanding the history of the main disk of the LMC is fundamental to our knowledge of the LMC's evolution and to the interpretation of properties of blue galaxies seen in the distant universe at substantial lookback times (Ellis 1997). If we cannot decode the history of star formation in the nearby LMC, then we must be concerned about the credibility of our analyses of distant galaxies (Tolstoy 1998).

A variety of measurements can be used to measure star formation rates $(S F R)$ and histories $(S F H)$ in galaxies. Integrated $S F R s$ are determined, for example, from $\mathrm{H} \alpha$, far infrared, or ultraviolet luminosities (Kennicutt 1998), 
while the $S F H$ depend on measures of ratios between younger and older stars, as measured by broad band colors or $\mathrm{H} \alpha$ emission equivalent widths. The LMC has the advantage that individual stars can be observed; thus we can apply the techniques of "Galactic astronomy" to another system. By observing the distributions of stars as functions of age, mass, and metallicity, detailed information about the $S F H$ of the LMC over cosmic times scales is obtained (Hodge 1989). In this paper we consider some aspects of this problem from the perspective of modern ground-based and WFPC2 digital photometry of stars in the LMC.

\section{Measuring Resolved Stars}

Images of any object suffer from a variety of defects and as a result we cannot perfectly measure characteristics of the target. Crowding in the dense star fields of even a nearby galaxy like the LMC results in overlaps between stars; faint objects are lost and the accuracies of colors and magnitudes are reduced. In the common case of confusion between a star on the red giant branch (RGB) and one on the main sequence (MS), the resulting image would land in the gap between the MS and RGB on an observed color-magnitude diagram (CMD). The extreme example of this effect occurs in physical binary stars, where the components cannot be resolved. An equal mass the binary has the color of a single star but is $0.75 \mathrm{mag}$ too bright. With increasing mass ratio the binary becomes redder in color and closer in brightness to the primary. Fortunately these effects can be handled statistically. However, close binary evolution also produces stars with unusual properties that are not included in single star evolutionary models (e.g., blue stragglers in star clusters).

Additional uncertainties can arise in even excellent data from a variety of factors. Background galaxies are often compact and confused with stars, especially at very faint magnitudes. Photometric transformations to standard systems have limits to their precision for observed stars and for the models. Even when these are very accurate, they may suffer from non-unique conversions between observables and stellar parameters, as in age-metallicity degeneracies on the RGB (Da Costa 1998). Observed colors and magnitudes are also influenced by dust within the LMC and in the Galactic foreground.

Given these complexities in interpreting LMC CMDs, is this still a worthwhile enterprise? Yes, because this is one of the best approaches that we have; other methods based on integrated properties simply hide many of the difficulties (e.g., Hodge 1989; Tolstoy \& Saha 1996; Grebel 1997; Mateo 1998).

Observational progress can still be made in improving the quality of CMDs. Data from WFPC2 have minimal crowding problems in most of the LMC; the WFPC2's 0.1 arcsec pixel is $0.02 \mathrm{pc}$ and it can reach to V 27 (or $M_{V} \approx 8$; corresponding to $0.6 M_{\odot}$ ) at the LMC. Multicolor measurements, particularly in the blue and infrared, allow effects of extinction to be traced (Harris et al. 1997; Romaniello 1998), as do the high angular resolution HI maps of Kim (1998). Intermediate band filter observations can play an important role in giving a first look at metal abundances in large populations of stars (e.g., Ardeberg et al. 1997; Cole et al. 1999). Figure 1 shows an example of a modern LMC disk field CMD derived from observations with WFPC2. 

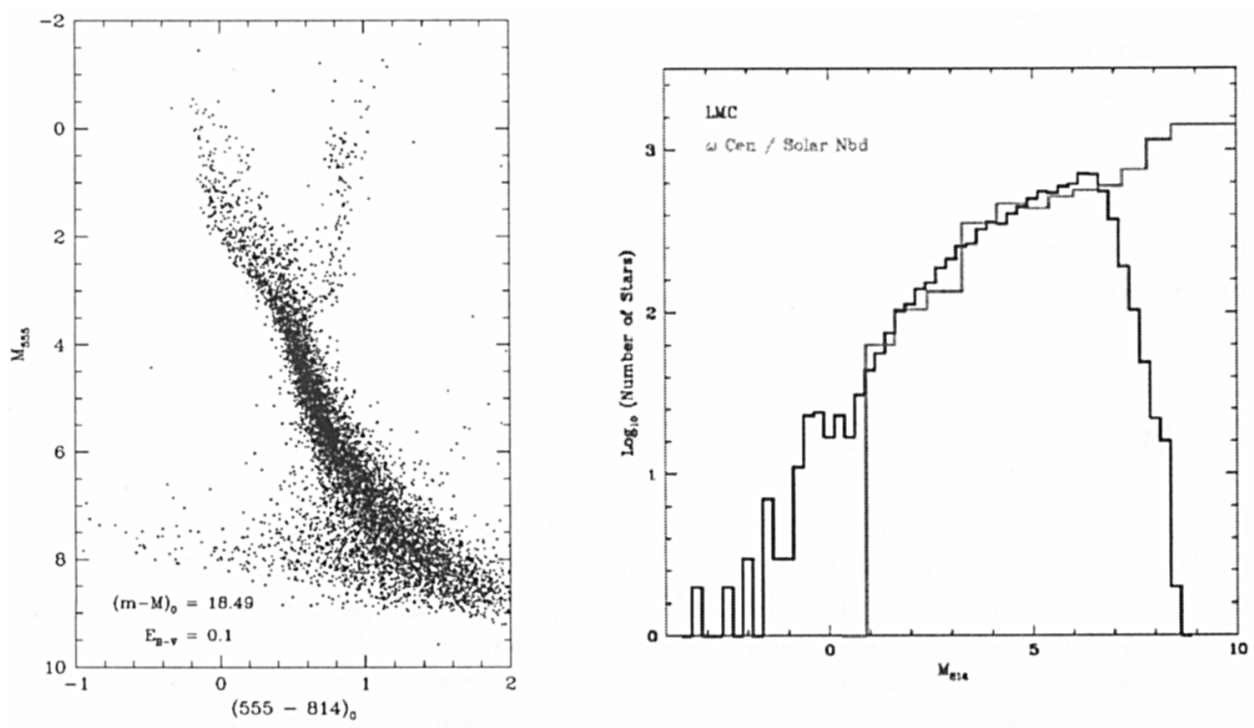

Figure 1. Left: mean disk LMC CMD in the WFPC2 VI-bands from the three fields studied in Geha et al. (1998). Note the MS band, lower RGB, red clump at $\mathrm{M}_{F 555 W} \approx 0.5$, and subgiants between the RGB and MS for older stars. Right: The LMC LF compared with that of Galactic star clusters (Elson et al. 1995; von Hippel et al. 1996).

\section{Interpretation of LMC CMDs}

\subsection{Stellar Luminosity Functions}

The numerical dominance of stars on the main sequence (MS) is obvious in Figure 1. Stellar luminosity functions (LF) can therefore be measured with unusual ease from such observations; there are no serious effects from variations in sample volumes with magnitude, as in Galactic studies (Mighell \& Butcher 1992). Even though we observe only small patches of the LMC disk with each WFPC2 pointing, orbital phase mixing randomizes the locations of disk stars in a few LMC rotation periods or $\sim 1$ Gyr (Gallagher et al. 1996).

Results for MS luminosity functions in disk fields are given by Holtzman et al. $(1997 ;$ H97) and Geha et al (1998; G98). These papers show that the MS LF in the LMC is similar to that in the solar neighborhood, or to those of metal-rich globular clusters below the MS turnoff (Figure 1). G98 also demonstrate that the LMC LF is relatively constant with position in the LMC disk.

Interpreting the MS LF depends on both the $S F H$ and IMF (e.g., Scalo 1986). Thus it is always possible to trade between the form of the IMF and the $S F H$ to some degree. G98, however, showed that for a Salpeter IMF $(n(m) d m \propto$ $m^{-2.35} \mathrm{dm}$ ) over the observed range of stellar masses, models of the LMC $S F H$ with highly enhanced recent $S F R$ s (e.g., the models suggested by Bertelli et al. (1992) or derived from the birthrates of star clusters) have too many intermediate age stars. Good evidence exists that the recent $S F H$ for the LMC field stellar 
population differs from that for compact star clusters, which probably survive as bound objects for long time periods; the enhancement in field SFR in the past 2-4 Gyr is less extreme than that implied by the cluster formation rate (see also van den Bergh 1998).

G98 conclude their study with an acceptable $S F H$ model which has constant $S F R$ for $\sim 2-10$ Gyr before the present, a modest enhancement in the $S F R$ during galaxy formation $\sim 10-12 \mathrm{Gyr}$ ago, and a factor of 3 increase in $S F R$ during the past 2 Gyr. Further tests of this LMC $S F H$ model await the larger sample of field stars observed with WFPC2 by Smecker-Hane et al. (1999), as well as better information on chemical element abundances in stars with intermediate and old ages.

\subsection{CMD Model Fitting}

Additional information beyond that contained in the MS LF can be derived by fitting the three dimensional distribution of stellar densities on a CMD (the Hess diagram). The observed LMC field star CMD is a sum of a large number of population components which can be characterized by the $S F R$ as a function of time and metallicity (Hodge 1989), where we begin with the probably realistic assumption that the IMF has remained approximately constant over time. An example of the complications introduced by such composite populations is provided by the structure of the RGB (e.g., Da Costa 1998; Gallagher et al. 1998); blue parts of an RGB can be made either by old, very metal-poor stars or younger, more massive stars. In a complex stellar population fits must be made to both the MS and RGB to distinguish between these options.

Numerical techniques for fitting CMDs are still being developed. A key point concerns the weighting of model fits. In the WFPC2 VI-band studies of LMC disk fields, the distributions of stars just below the least luminous MS turnoff, with $\mathrm{M}_{V} \approx 4-5$, tend to drive $S F H$ model fits to the entire $\mathrm{CMD}^{1}$. Thus we are in effect fitting only a modest range in stellar mass rather then the entire CMD. Techniques such as those of Tolstoy \& Saha (1996) allow a more global approach to CMD fitting, as do the numerical approaches of G98, Han (1998), and Hernandez et al. (1998).

All of these methods depend on the fidelity of the stellar evolution models, and on the correct choice of distance and extinction to the field being studied. The former point is currently a major issue. Evolution tracks from stellar models from the various groups disagree in detail, a natural result of differing choices, e.g., for convective core overshoot or mixing. In addition the current models often exclude "second-order" effects which exist in real stellar populations, such as stellar rotation; these influence, e.g., the MS band (Gallagher et al. 1996).

Of course when we attempt to fit stars in different evolutionary phases additional problems emerge. For example, a variety of studies suggest that colors of the RGB in intermediate age and metallicity stellar models are too red in all but the most recent stellar evolution models (Gallagher et al. 1996; Elson et al. 1997; Girardi et al. 1999). Thus to get a fit to the observed RGB, the $S F H$ models must include old, metal-poor populations that are not necessarily

\footnotetext{
${ }^{1}$ This occurs because fitting weights scale roughly as the color error divided by the square root
} of the number of stars, and this peaks just below the last MS turnoff in WFPC2 data; Fig. 1. 
present, as indicated by the location and stellar densities at low luminosity MS turn offs. But with the increasing sophistication of stellar evolution and atmosphere models and large amounts of new stellar data from observations of LMC star clusters with the HST and soon with the 8-m VLT and Gemini Telescopes, the reliability of the LMC CMD fitting process will rapidly improve.

\section{Discussion}

In addition to the deep studies emphasized in this paper, we will also benefit from shallower, wide angle surveys in the optical and infrared (Zaritsky et al. 1997); these are yielding nearly complete information on more luminous stars and patterns of interstellar dust obscuration and reddening within the LMC.

Even so our understanding of the $S F H$ of the LMC disk remains a work in progress. However, several key points already stand out:

1. The stellar LF and mid-mass range $\left(0.7-2 M_{\odot}\right)$ IMF for the disk of the LMC are similar to their Galactic equivalents. The average stellar IMF in galactic disks seems to be roughly constant over a wide range of conditions.

2. Building from Butcher's (1977) initial explorations, we are beginning to obtain reasonable empirical models for the $S F H$ of the LMC. While these are consistent with those derived from the chemical evolution of the LMC (Dopita 1999; Pagel \& Tautvaišienè 1998), they do not agree with standard models for galactic evolution. The LMC appears to have experienced neither smooth evolution with constant SFRs nor extreme, short duration starbursts. Three major epochs of star formation are found during which average SFRs were roughly constant, albeit at different levels. The physical origins of this behavior are not known, although interactions with the Milky Way, SMC, and their surrounding interstellar material remain prime suspects in this unsolved case.

3. The recent $S F H$ of the stellar field in the LMC disk shows less extreme time variations than the birth rate of compact star clusters (G98; van den Bergh 1998).

We now have exciting opportunities to extend LMC $S F H$ studies to a wider range of issues. We still need to measure how the ratio of intermediateto-old age stars varies with radius in the LMC disk and to see if the bar has a distinct evolutionary history. Preliminary investigations suggest that the LMC, like several other galaxies with dwarf-like structures, has flatter radial gradients in its older stellar components. Thus it would have been a diffuse object in its youth (van den Bergh 1991), unlike the compact blue faint galaxies. This leaves the possible formation of the bar as the last likely repository for a stellar population that could have formed in an intense starburst. We will no doubt have interesting new results on the $S F H$ for the next LMC Symposium.

Acknowledgments. We thank many of our colleagues for discussions of the Magellanic Clouds and related topics as well as sharing their results: R. Dohm-Palmer, R. Elson, E. Grebel, P. Hodge, D. Hunter, P. Linde, P. Massey, J. Mould, E. Skillman, E. Tolstoy, B. Wakker, and especially Sidney van den 
Bergh. JSG, AAC, and JAH were supported in part by NASA under contract NAS 7-1260 to JPL for the WFPC2 Investigation Definition Team.

\section{References}

Ardeberg, A., Gufstafsson, B., Linde, P., \& Nissen, P. E. 1997, A\&A, 322, L13 Bertelli, G. Mateo, M., Chiosi, C., \& Bressan, A. 1992, ApJ, 388, 400

Butcher, H. 1977, ApJ, 216, 372

Cole, A. A., Smecker-Hane, T., \& Gallagher, J. S. 1999, in this volume

Da Costa, G. S. 1998, in Stellar Astrophysics for the Local Group, eds. A. Aparicio, A. Herrero, F. Sánchez (Cambridge, Cambrdige University Press), p. 351

Dopita, M. 1999, in this volume

Ellis, R. C. 1997, ARA\&A, 35, 389

Elson, R. A., Gilmore, G. F., Santiago, B. X., \& Casertano, S. 1995, AJ, 110, 682

Elson, R., Gilmore, G. F., \& Santiago, B. X. 1997, MNRAS, 295, 860

Gallagher, J. S., et al. 1996, ApJ, 466, 732

Gallagher, J. S., et al. 1998, AJ, 115, 1869.

Geha, M.C., et al. 1998, AJ, 115, 1045

Girardi, L., Bressan, A., Bertelli, G., \& Chiosi, C. 1999, in preparation

Grebel, E. 1997, RvMA, 10, 29

Han, M. 1998, private communication

Harris, J., Zaritsky, D., \& Thompson, I. 1997, AJ, 114, 1933

Hernandez, X., Valls-Gabaud, D., \& Gilmore, G. 1998, MNRAS, 304, 705

Hodge, P. 1989, ARA\&A, 27, 139

Holtzman, J., et al. 1997, AJ, 113, 656

Kennicutt, R. C. 1998, ARA\&A, 36, 189

Kim, S. 1998, Ph.D. Thesis, The Australian National University

Mateo, M. 1998, ARAA, 36, 435

Mighell, K. J. \& Butcher, H. R. 1992, A\&A, 255, 26

Pagel, B.E.J., \& Tautvaišienè 1998, MNRAS, 299, 535

Romaniello, M. 1998, Ph.D. thesis, University of Pisa

Scalo, J. 1986, FCPh, 11, 1

Smecker-Hane, T., et al. 1999, in this volume.

Tolstoy, E. 1998, in Dwarf Galaxies and Cosmology, eds. T. X. Thuan et al., p. 213

Tolstoy, E., \& Saha, A. 1996, ApJ, 462, 672

van den Bergh, S. 1991, ApJ, 369, 1

van den Bergh, S. 1998, ApJ, 507, L39

von Hippel, T., et al. 1996, AJ, 112, 192

Westerlund, B. 1997, The Magellanic Clouds, (Cambridge, Cambridge U. Press) 
Zaristky, D., Harris, J., \& Thompson, I. 1997, AJ 114, 1002

\section{Discussion}

Carme Gallart: I would like to point out that the comparison of isochrones with CMD's of complex (not single age) stellar systems can be quite misleading because a) the $\mathrm{Z}$ in the isochrones are discrete and not necessarily adequate for the particular set of data (small changes in $Z$ lead to large changes in posiiton in the CMD) and b) the isochrones don't give information on the lifetime of the stars along it.

Gallagher: I think we agree - isochrones provide a means for a first look at a data set, but are not a proper tool for fitting galactic Hess diagrams. 